\title{
Inter-Domain Integration of Services and Service Management
}

\author{
David Lewis, Thanassis Tiropanis, Alistair McEwan \\ Department of Computer Science, \\ University College London \\ D.Lewis,T.Tiropanis,A.McEwan@cs.ucl.ac.uk
}

\author{
Cliff Redmond, Vincent Wade \\ Department of Computer Science, \\ Trinity College Dublin
}

Cliff.Redmond, Vincent.Wade@cs.tcd.ie

\author{
Ralf Bracht \\ IBM, European Networking Centre, \\ R.Bracht@heidelbg.ibm.com
}

\begin{abstract}
The evolution of the global telecommunications industry into an open services market presents developers of telecommunication service and management systems with many new challenges. Increased competition, complex service provision chains and integrated service offerings require effective techniques for the rapid integration of service and management systems over multiple organisational domains. These integration issues have been examined in the ACTS project Prospect by developing a working set of integrated, managed telecommunications services for a user trial. This paper presents the initial results of this work detailing the technologies and standards used, the architectural approach taken and the application of this approach to specific services.
\end{abstract}

Keywords: Inter-domain service management, TINA Service Architecture, reusable service components

\section{Introduction}

The emergence of an open market in telecommunications services is occurring globally. Competition in this market-place will increase pressure on service providers to develop and deploy services of increasing functionality and diversity in shorter time-frames. This presents a major challenge for the telecommunications industry, i.e., to accelerate the service life-cycle so that new services can be developed and deployed at a faster rate. This will enable services to evolve fast enough to meet changing customer demands competitively. In such a competitive market, services will be difficult to offer as a vertically integrated system developed by one provider; instead services will have to integrate with other services and service components operated by different providers. This co-operation may be motivated by providers' different market positions, e.g. allowing specialised providers to use the greater geographical coverage or resource capacity of a larger provider, or allowing customised services to be offered by combinations of separate providers' offerings.

Combining service offerings in this way requires the integration of service software components as well as the integration of the management components that manage these services. One approach to such integration could be accessing service and management functionality via industry agreed interfaces. This provides opportunities for third party service software developers to provide "off the shelf" reusable components. Service management components may be suitable for such development, since they encompass relatively stable systems such as the underlying network management systems and legacy customer and billing databases. This approach may however prove problematic where competitive pressures force the pace of change 
beyond that at which industrial agreements can be readily made. In this case common management and service components intended for reuse in different service systems will require appropriate flexibility in order to suit the requirements for new services as they emerge.

This paper presents the practical results of the ACTS project Prospect in examining the integration of telecommunications services and management in the open service market. In such a market there is the possibility of a wide range of new service providers as well as complex relationships between them and their customers. The particular business situation adopted in Prospect (see figure 1) is intended to provide a reasonably complex business scenario to generate realistic service integration problems. This scenario is based on a provider of educational courses that offers a Tele-Education Service (TES) to its customers. This service is an integration of three examples of a Multimedia Tele-Service (MMTS, i.e. a distributed, multimedia telecommunications service); a Hyper-Media (HM) service, a Multimedia Conferencing (MMC) service and a Web-Store (WS) service, each provided to the TES by a separate provider organisation. In addition the TES provider subscribes to a general purpose Virtual Private Network (VPN) service that it uses to provide broadband network connectivity between itself, its customers and the MMTS providers. The VPN provider further extends the service chain by using the ATM Virtual Path management service offered by a public network provider, delivering international links between the connected customer and provider sites.

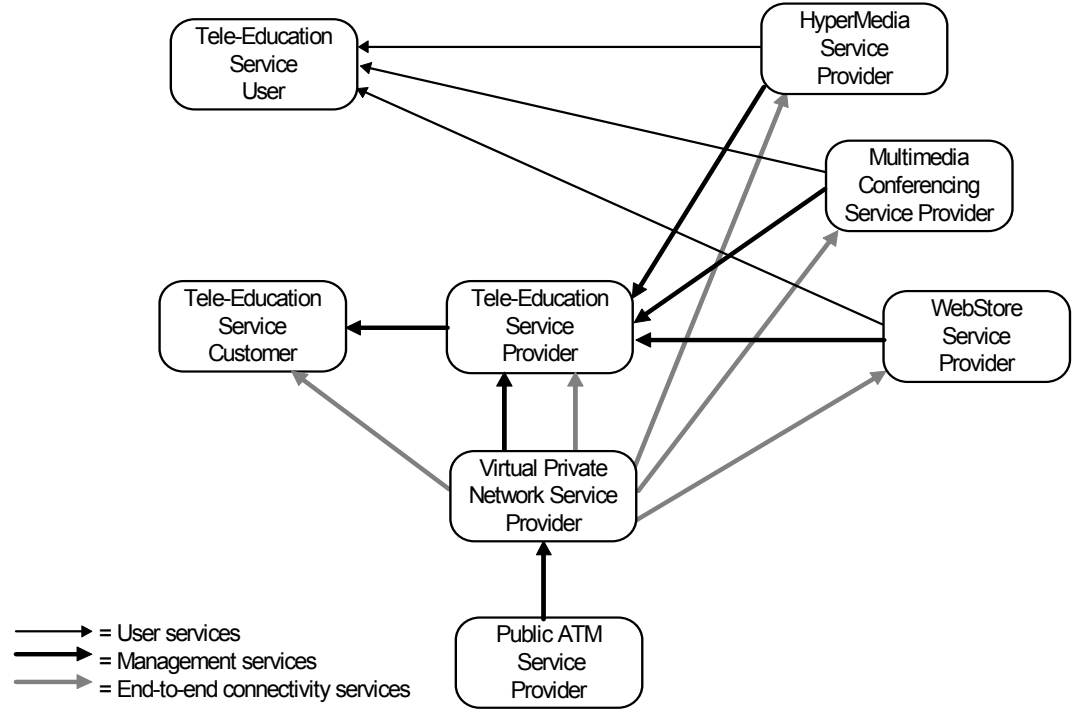

Figure 1: Prospect Stakeholder Model

The following section presents some of the contemporary technologies and architectures adopted for implementing this business model. Section 3 describes the overall approach to service and management integration in Prospect, and provides details on some specific design areas. The future work planned in this project is then outlined before concluding. The actual analysis and design methodology is not given here but is discussed in more detail in [Wade]. 


\section{Architectures and Technologies}

The aim of Prospect's investigations into the integration of telecommunication services and management was to develop a pragmatic approach, both to facilitate the development of a working trial and to ensure that the guidelines developed would be relevant to both existing and evolving technologies, architectures and services. The selection of the approaches adopted is summarised below.

\subsection{Architectures: TMN and TINA}

The Telecommunications Information Networking Architecture (TINA) Consortium has been specifically addressing the area of telecommunications services and their management. For this, TINA has developed a service architecture [TINA-SA], a TMN-based management architecture [TINA-MA] and a network architecture (influenced by ATM Forum and TMN network models). These architectures provide a consistent, detailed model for the integration of service, management and network components.

For Prospect, the integrated service and management model of the TINA Service Architecture (SA) was selected as the basis of the TES and MMTS providers' systems as described in the next section. TINA's integration of the network architecture with the service and management architectures was not, however, adopted, since it assumes a call-based model that cannot be straightforwardly resolved with the connectionless communication model used by the Internet applications used in Prospect (see section 2.3). The information technology approach taken in TINA is based on a distributed processing environment (DPE) defined in the TINA computing architecture [TINA-DPE]. However due to the lack of any DPE implementations, alternative approaches were adopted as discussed in the next section.

\subsection{Software Integration Technologies: CORBA and Java}

Recent development in distributed systems technologies have been led by the Object Management Group [OMA], and in particular their definition of the CORBA-2 standard. CORBA 2 allows for the specification of object interfaces and their abstract location in a (virtual) distributed environment. Object interface definition is separated from object implementation, thus allowing components of a CORBA 2 compliant object network to use different (possibly proprietary) implementation languages. The freedom this imparts to the developers of components, intended for integration into large systems, ideally places this technology for use in the open services market. In Prospect CORBA was used primarily for implementing computational objects derived from the TINA SA. These objects typically had multiple interfaces, which were mapped onto individual CORBA objects.

The user interface systems of the Prospect system has some specific requirements centred around the close integration of components and portability, i.e. the user interfaces have to integrate the interfaces offered by the different services being made available to users in an apparently seamless way on both UNIX and Win32 platforms. The definition of an IDL mapping for Java in the CORBA 2 standard allows for this language to be used to implement desktop service client components for users. The portability of Java and the well defined inter-applet communication interface meant 
that this was the most viable solution. In addition, Java provided a path to the integration of the user interface components with WWW browsers, either as Java applets or as Java implementations of Netscape plug-ins, and to the dynamic distribution of service client component software. The Java AWT library also was used to provide integrated, portable graphical user interfaces.

\subsection{Network Technologies: Internet and ATM}

The focus of Prospect is on an open market for digital broadband services. ATM is often assumed to be the primary technology to provide such services, yet the lack of commercial, switched wide area ATM services and the absence of any killer applications is delaying its widespread adoption. In comparison, Internet technology is the major solution for open digital communications today, with a huge growth in commercial Internet service providers and a wide range of applications and services being available. Two of the major developing strands of Internet services are represented in Prospect:

- The $M-B O N E$, though still largely a non-commercial trial service, has shown the feasibility of real time, multiway, multimedia communications. This technology is easily scaleable from small to large groups of communicating individuals and makes optimum use of network resources.

- The World Wide Web has been largely responsible for the recent explosive growth in the use and extent of the Internet, as well as providing the motivation for the adoption of Intranet systems in many corporate networks. The ease of providing information, the free availability of browsers and the use of a simple communications protocols has enabled providers to develop and deploy new services in an open way, thus allowing a wide range of commercial services to be offered over telecommunications networks.

The Internet community is now starting to deploy mechanisms [RSVP] for providing the kind of range of quality of service guarantees that makes ATM attractive for integrated services. The resulting possibilities for convergence of IP and ATM technologies have already been commented on [Crowcroft], and interest in new technologies such as IP switching, point to a combined IP-ATM approach as being a good basis for the integration of services in the open service market. Prospect has therefore elected to use existing IP applications between hosts on mixed technology LANs, communicating via routers interconnected over a backbone of campus and wide area ATM networks. Such a configuration provides paths to both the parallel development of IP and ATM services and the convergence of these technologies. It is also typical of current ATM usage and therefore provides a realistic and practical basis for broadband user trials.

The required IP connectivity was provided in a generic way by the VPN service. This supports the assembly of a multi-domain Intranet (sometimes termed an "Extranet"), in this case connecting customer, TES provider and MMTS provider LANs. This was controlled in a TMN-structured management plane, separate to the control of the services themselves, i.e. the service sessions did not interact directly with communication sessions in the way prescribed in TINA. Issues of network resource and connection management in Prospect are not discussed further in this paper but are addressed in more detail in [Bjerring]. 


\section{Integration Model}

This section presents the approach taken for the integration of both service and management components in developing integrated digital services in Prospect. The development work reported here has attempted to simulate software development in the open services market, in particular by the development of common reusable components that can be readily integrated into the service and management systems of customers and providers for specific services.

\subsection{Common Service and Management Components}

The enterprise model contains several organisations providing different types of services, however many of the user and management activities that need to be performed by the systems used by customers and providers are similar, and therefore lend themselves to common solutions. This was appropriate for the TES and MMTS providers who all have to solve similar problems with the control and accounting of end user access to their services. The approach taken to these problems involved the development of common component interfaces and implementations that were reused in the different systems of the various service providers.

The TINA SA integrates the end user's access to and interaction with a telecommunications service with the management of service-related subscription and accounting information. This architecture identifies areas that are regarded as service specific, i.e. those that will be different for different service offerings, and those which are service independent, i.e. those that will remain constant over different service implementations. In this way, the SA model provides a core set of common inter-operable interfaces between its components, as well as a common semantic model around which services can be developed, both of which aid in the integration of separate services. The TINA SA was therefore deemed well suited as a basis for common service and management components in Prospect. The version of the SA publicly available when this work was conducted assumed, however, that all services were supplied by a single provider. This architecture therefore had to be augmented in order meet the multi-domain requirements of the Prospect business model.

The major common components of the Prospect design model derived from the TINA SA are; the integration of service control in the user and provider domains, termed the Desktop Service Integration (DSI) component; the Subscription Management component and the Accounting Management component.

The DSI component is based on the TINA separation of issues related to user access to services and issues related to usage and control of the service. These are modelled in TINA as separate access and service sessions, but augmented in Prospect to operate with existing desktop technologies and to use Internet protocols for service data flow, rather than an explicitly controlled communications session as prescribed in TINA. The aim was to provide a single model that each of the providers could use, but which could be easily integrated at the desktop in a way that would render the multi-provider composition of the services transparent to the end user. This required a clear distinction between the parts of the model which were service independent and could therefore be used directly by each service provider, and parts of the model that were service specific, and were thus expected to be augmented by the individual service providers to accommodate functionality particular to their service. 


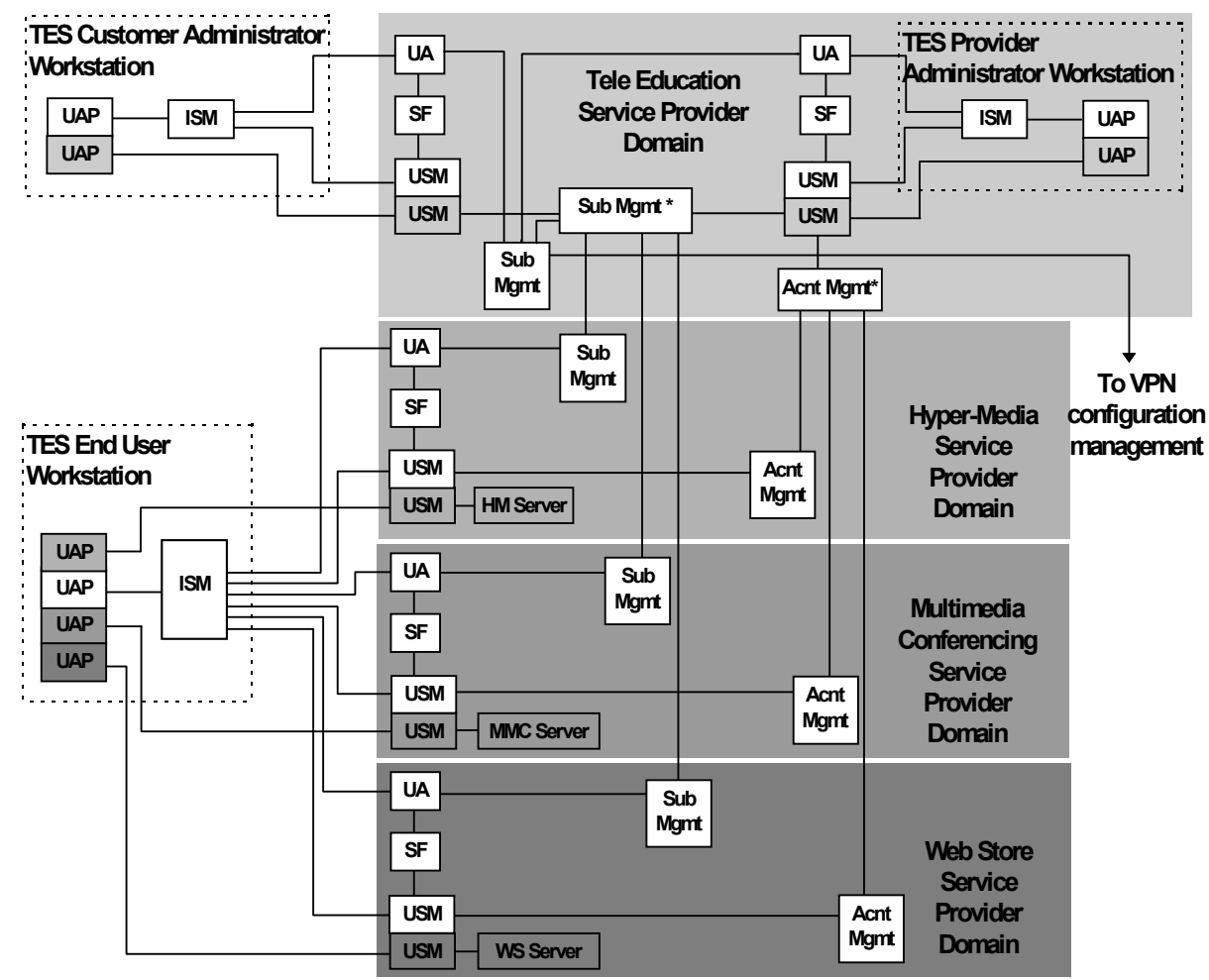

Figure 2: Integration of Service independent and service specific components

In the user domain the DSI model modifies the TINA SA to support the integration of existing applications, adopting a component-based approach using Java applets integrated into a WWW browser which together form the user application (UAP) component of the model. Also in the user domain a separate object, named the integrated session manager (ISM) mediated all the service independent interactions between the user and provider domain. This was based on the TINA notion of a Generic Session End Point but with additional support for hierarchical session relationships, thus supporting the composition of service sessions from different providers, into what appears to the user to be a single service session.

In the provider domain the TINA SA concepts of a User Agent (UA), Service Factory (SF) and User Service Session Manager (USM) have been adopted, providing the service independent components that allow the user domain components to interact correctly with the Subscription and Accounting Management components. The TINA service session concept has however been simplified by removing the requirements for group session functionality. Where needed, this functionality is provided by service specific components, e.g. multicast groups in the MMC service. The UA mediates between the user and the Subscription Management component of the provider's system, relaying the services the user may access and allowing the user to initiate a session in one of those services. The SF is then used by the UA to create a USM that allows the user to interact with a service session. The TINA SA service session interactions, e.g. initiate, terminate, suspend and resume, are handled by the 
service independent parts of the USM, including logging them with the usage metering parts of the Accounting Management component. Other service specific interactions need to be implemented by the service provider responsible, as extensions to the USM and corresponding UAP components.

The Subscription Management component allows for the authorisation and barring of users to specific services, and the addition and removal of network sites supporting these users. Parts of the Subscription Management component are identified as areas for service specific specialisation; for instance the subscription registrar (SubRgs) component, which performs service specific operations on the basis of service independent ones it receives. The Accounting Management components allows service usage monitoring through interactions with a USM, charging based on tariffs agreed for a subscription, and bill generation based on these charges and a customer specific tariff plan which specifies discounts, payment schedules etc..

Figure 2 shows how the various service independent components (white boxes) and service specific components (shaded boxes) have been combined in integrating the service and management systems of the TES and MMTS providers. Within the project, these common components were implemented by one set of partners, and then reused by other partners in the implementation of the specific service provider systems they will operate. This simulated, to an extent, the development environment of the open service market which provided a means of gaining direct experience of some of the problems involved. All the objects shown in figure 2 were implemented on a single CORBA 2.0 compliant platform, i.e. Orbix, OrbixWeb and OrbixNames, to avoid any IIOP interoperability problems. Objects were actually implemented in either $\mathrm{C}++$ or Java. The following sections describe in more detail how common components were used for specific services.

\subsection{Integration of the Tele-education Service}

The TES system is a good example of how a service can be constructed from an integration of existing services and common, reusable service and management software components. The integration for this service offering can be decomposed into the integration of the user services and the integration of management services.

The integration of user services was performed in the user application. This was based on a WWW browser that integrated Java applets which performed the service independent UAP functions of the DSI components as well as applets supplied by the MMTS providers to perform their service specific interactions. The problem of integrating the services was therefore reduced to that of defining inter-applet communications. This enabled the TES UAP developer to concentrate on the look and feel of the user interface, and the composition of the MMTS in delivering the TES to the user, rather than how the UAP interacted with the different MMTS providers' systems.

The development of the TES management system involved both the development of provider domain management systems and mechanisms for the TES administrator and TES customers to interact with these systems. The TES needs to co-ordinate its management with that of the subcontracted MMTS and VPN providers. For subscription management this required propagating operations such as the activation and deactivation of service profiles and the authorisation and barring of end-users, to 
the Subscription Management components of the MMTS provider. This was done by developing a wrapper (SubMgr* in figures 2) for the TES provider's common Subscription Management component, that forwarded the relevant operations to the identical common components in the MMTS providers' domains. In addition the SubRgs CO of the TES was modified to perform the correct configuration management operations when a customer site was added or removed, i.e. to use the VPN provider's configuration management services to connect to this site. For accounting management the interactions with sub-providers was limited to the collection of billing information from the common Accounting Management components used by each MMTS provider.

The customer and provider management UAPs were based the common DSI component. For the provider the UAP and the USM were extended to provide the user with access to subscription and accounting management information as well as management functions controlling how this information is propagated to specific subprovider systems. For the customer the UAP and USM provided a subset of these operations, allowing the customer access only to specific operations related to their particular service subscriptions.

\subsection{Integration of the Hyper-Media Service}

The Hyper-Media service is a WWW service modified to facilitate accounting management, subscription management and service customisation management facilities for service customers.

The management service allows the generation, storage and access of per-user data on the service provider side. This can be used to provide functionality that is currently dependent on the client software implementation, which restricts the user to using the same client to avail of the bookmarks and history lists they generate.

Service specific enhancements to the USM component were needed to simulate a session context for WWW applications, which offer an inherently connectionless service. The overall requirements for a generic, unified, access session, an allowance for the provision of security services in the future, and the concept of a service session meant that the current access and security mechanisms the WWW were not sufficient for our needs. A revised definition of a service session, as a context in which HTTP service requests are made, was found more useful for a service which is not connection oriented. Simulating a session gives benefits, i.e. a known amount of users, user barring and user identification, and the solution developed here requires no modification of common client software, i.e. no service specific UAP is required. The TINA service session concept is implemented using a combination of browser state and a proxy mechanism. The proxy mechanism also ensures that each user accesses a document using a unique URL so organisational caching (which can occur in a organisational firewall application-level proxy cache) does not affect the per-user charge. The de facto support of a browser side state mechanism (Netscape 'Cookies') in current HTTP implementations lent itself for use as an application layer request context. This means that a conceptual session can exist in the application layer protocol interactions between a client and server.

The session mechanism works by initialising the client with a URL which, when accessed, creates a proxy for that user in the provider domain. Following a redirect, 
subsequent interactions to the service can only be made through this proxy. Usage information generated by the modified server is forwarded to the service independent metering component through a component that substitutes the user ID, which is originally passed to the service in the URL, for the 'Cookie' being used as the session identifier.

\section{Further Work}

The work described here was performed as an initial step in Prospect's on-going examination of key issues in multi-domain service and management integration. The TINA service architecture adopted in the model described here assumes a single service provider context, and therefore required extensions to handle the multidomain situation assumed in Prospect. However TINA-C has recently refined the service architecture to provide support for multiple providers, and alignment of the current Prospect model with this will be attempted. In addition, a major feature of the TINA service architecture is its built-in support for mobility, the exercising of which will be an important theme of future Prospect work.

The introduction of RSVP based services in the Internet, and its integration with applications and management services, particularly accounting, is also a key component to placing this work in a relevant business context, and is therefore a subject for further work. The integration of security is also a key aspect of system development and integration in the open services market. Though this work does not aim specifically to implement security mechanisms, it will attempt to integrate existing and emerging mechanisms, e.g. SecuDE, to assess their impact on practical service development.

\section{Conclusion}

This work provides an example of how the definition of common, reusable components aids in the rapid development of integrated telecommunications services. The availability of implementations of such components eases the development of individual services by providing common, well understood functionality "off the shelf". Care must be taken however in clearly defining what parts of a reusable component are intended to be used as-is, i.e., the service independent parts, from those that must be modified by its users to integrate into the service, i.e. the service specific parts. The widespread use of common components could also ease the task of developing integrated services, since the common parts can be designed explicitly to support such integration as demonstrated by the Prospect management components.

Though this project does not aim to be TINA compliant, the TINA service architecture was found to provide a practical framework for controlling and managing multimedia service offerings based on IP technology. Not all aspects of the TINA SA were used however, with communication session management and group session functionality being omitted. CORBA was found to provide a suitable basis for implementing TINA-based components, though fuller use of CORBA Common Object Services, e.g. the life-cycle service, is required. From this work, it also appears that a combination of WWW, Java and CORBA provides a practical approach to presenting integrated services to the user. However a suitable security model for 
applets that need to communicate with multiple remote objects needs to be supported in WWW browsers.

\section{Acknowledgements}

The work presented in the paper was conducted with partial funding of the European Commission under the Prospect project (number AC052). The authors would also like to thank all members of the Prospect team for their collaboration in this work and Patrick McLoughlin of Broadcom for his comments.

\section{References}

[Crowcroft] Crowcroft, J., Wang, Z., Smith, A., Adams, J., A Rough Comparison of the IETF and ATM Service Models, IEEE Networks, Vol, 9, No. 6, July 1995

[Bjerring] Bjerring, L., Lewis, D., Thorarensen, I., An Inter-domain Virtual Private Network Management Service, IEEE/IFIP 1996 Network Operations and Management Symposium, Vol 1, pp115-123, IEEE, NJ, 1996

[OMA] Object Management Architecture Guide, ed. R.M. Soley, OMG Document Number 92.11.1, Revision 2.0 Second Edition, Object Management Group, 1992

[RSVP] Zhang, L., Deering, S., Estrin, D., Shenker, S., Zappala, D., RSVP, a new resource ReSerVation protocol, IEEE Network, vol. 7, no. 5, September/October 1993

[TINA-DPE] Graubmann, P., Mercouroff, N., Engineering modelling Concepts (DPE Architecture), TINA Baseline Document TB_NS.005_2.0_94, December 1994

[TINA-MA] de la Fuente, L., A., Walles, T., Management Architecture, TINA Baseline Document TB GN.010 2.0 94, December 1994

[TINA-SA] Bernt, H., Kim, C., Kim, L., et al., Service Architecture, TINA Baseline Doc. No. TB_MDC.012_2.0-94, TINA-C, March 1994

[Wade] Wade, V., Sheppard, M., Tschichholz, M., Hall, J., Lewis, D., A Methodology for Developing Integrated Multi-domain Service Management Systems, Proceeding of IS\&N ’97, Como, Italy, April 1997 